\title{
Num open \$-3 fatty acid supplement use in the 45 and Up Study Cohort
}

\author{
Jon Adams, ${ }^{1}$ David Sibbritt, ${ }^{1}$ Chi-Wai Lui, ${ }^{2}$ Alex Broom, ${ }^{3}$ Jonathan Wardle ${ }^{1}$
}

To cite: Adams J, Sibbritt D, Lui C-W, et al. $\Omega-3$ fatty acid supplement use

in the 45 and Up Study Cohort. BMJ Open 2013;3: e002292. doi:10.1136/ bmjopen-2012-002292

- Prepublication history for this paper are available online. To view these files please visit the journal online (http://dx.doi.org/10.1136/ bmjopen-2012-002292).

Received 1 November 2012 Revised 27 February 2013 Accepted 11 March 2013

This final article is available for use under the terms of the Creative Commons Attribution Non-Commercial 2.0 Licence; see http://bmjopen.bmj.com

${ }^{1}$ Faculty of Health, University of Technology Sydney, Sydney, Australia

${ }^{2}$ School of Population Health, University of Queensland, Brisbane, Australia ${ }^{3}$ School of Social Science, University of Queensland, Brisbane, Australia

\section{Correspondence to} Professor Jon Adams: jon.adams@uts.edu.au

\section{ABSTRACT}

Objective: There has been a dramatic increase in the use of dietary supplements in Western societies over the past decades. Our understanding of the prevalence of $\Omega-3$ fatty acid supplement consumption is of significance for future nutrition planning, health promotion and care delivery. However, we know little about $\Omega$-3 fatty acid supplement consumption or users. This paper, drawing upon the largest dataset with regard to $\Omega-3$ fatty acid supplement use $(n=266848)$, examines the use and users of this supplement among a large sample of older Australians living in New South Wales.

Design: A cross-sectional study. Data were analysed from the 45 and Up Study, the largest study of healthy ageing ever undertaken in the Southern Hemisphere.

Setting: New South Wales, Australia.

Participants: 266848 participants of the 45 and Up Study.

Primary and secondary outcome measures: Participants' use of $\Omega-3$, demographics (geographical location, marital status, education level, income and level of healthcare insurance) and health status (quality of life, history of smoking and alcohol consumption, health conditions) were measured.

Results: Of the 266848 participants, 32.6\% reported having taken $\Omega-3$ in the 4 weeks prior to the survey. Use of $\Omega-3$ fatty acid supplements was higher among men, non-smokers, non-to-mild (alcoholic) drinkers, residing in a major city, having higher income and private health insurance. Osteoarthritis, osteoporosis, high cholesterol and anxiety and/or depression were positively associated with $\Omega-3$ fatty acid supplement use, while cancer and high blood pressure were negatively associated with use of $\Omega-3$ fatty acid supplements.

Conclusions: This study, analysing data from the 45 and Up Study cohort, suggests that a considerable proportion of older Australians consume $\Omega-3$ fatty acid supplements. There is a need for primary healthcare practitioners to enquire with patients about this supplement use and for work to ensure provision of good-quality information for patients and providers with regard to $\Omega-3$ fatty acid products.

\section{INTRODUCTION}

There has been a dramatic increase in the use of dietary supplements in Western

\section{ARTICLE SUMMARY}

Article focus

- The use of dietary supplements has increased rapidly in Western societies over the past few decades.

- Our study examines the use of $\Omega-3$ fatty acid (w3 FA) supplements among older Australians, drawing upon the largest study of healthy ageing undertaken in the Southern Hemisphere $(\mathrm{n}=266$ 848).

Key messages

- A considerable proportion of older Australians living in New South Wales (32.6\%) report using w3 FA supplements.

- People with osteoarthritis, osteoporosis, high cholesterol and anxiety and/or depression were more likely to use w3 FA supplements, while people with cancer and high blood pressure were less likely to use w3 FA supplements.

- There is a need to ensure provision of goodquality information for patients and providers with regard to w3 FA supplement products.

Strengths and limitations of this study

- Our study benefits from the use of the largest sample of Australians aged 45 years and above with regard to the consumption of w3 FA supplement.

- The interpretation of our findings is limited by the fact that the use of w3 FA supplement was self-reported by the participants and their answers may have been subject to recall bias.

industrialised societies over the past few decades. The proportion of American adults using at least one dietary supplement has increased from $42 \%$ to $53 \%$ between 1994 and 2006. ${ }^{1}$ Research also shows that the use of dietary supplements is common in European countries such as Denmark (66\% of men and $51 \%$ of women) and the UK (48\% of women and $36 \%$ of men). ${ }^{2}$

In 2007, a national US survey identified

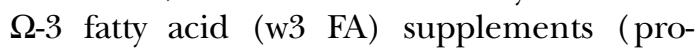
ducts containing docosahexaenoic acid and eicosapentaenoic acid products) as the first (for adults) and second (for children) most commonly used natural (non-vitamin, 
non-mineral) product in America. ${ }^{3}$ In Australia, a substantial proportion of general practitioners (GPs) $(80.3 \%)$ and community pharmacists $(90.2 \%)$ recommend $\Omega-3$ to patients ${ }^{4}$ and findings from this Australian research also identify w3 FA supplement as among the top five complementary and alternative medicines about which healthcare professionals $(71.6 \%$ of GP and $71.7 \%$ of pharmacists) desire better-quality information.

The clinical evidence base for w3 FA supplement use is varied depending on the specific condition. There is evidence that w3 FA supplements help in preventing or benefiting the outcomes of cardiovascular disease with some evidence suggesting a cholesterol-lowering effect. ${ }^{5-7}$ However, a meta-analysis study released in 2012 found no relation between the use of w3 FA supplements and reduced risk of major cardiovascular diseases. ${ }^{8}$ At present, the National Heart Foundation of Australia recommends all Australian adults consume about $500 \mathrm{mg}$ (or $1000 \mathrm{mg}$ for those who have documented coronary heart disease) of w3 FA per day. ${ }^{9}$

Beyond cardiovascular disease, existing research has found no evidence of a significant association between w3 FA supplement use and reducing cancer incidence. ${ }^{10}$ However, there is some evidence that w3 FA supplements may improve clinical, biological and quality-of-life parameters among patients with advanced cancer. ${ }^{11}$ There is currently conflicting or insufficient scientific evidence on the efficacy of w3 FA supplements regarding improvement of mental health disorders, ${ }^{12} 13$ asthma, ${ }^{14}$ cystic fibrosis, ${ }^{15}$ rheumatoid arthritis, inflammatory bowel disease and osteoporosis $^{16}$ and cognitive functions affected by aging, dementia and neurological diseases. ${ }^{17} 18$

Given the rise in ageing population and increasing public awareness of the importance of preventive health, ${ }^{19}$ knowledge about consumption of dietary supplements such as w3 FA is of significance for future health promotion and healthcare delivery. In response, this paper describes the findings of the first study to examine the use of w3 FA supplements in Australia. It aims to provide analysis of the prevalence and characteristics of $\Omega-3$ use among a large sample of Australians $(\mathrm{n}=266848)$ aged 45 years and above.

\section{METHOD}

Sample

This research utilised data collected through the 45 and Up Study, which is the largest study of healthy ageing conducted in the Southern Hemisphere and analyses of data from 266848 men and women aged 45 and above who reside in the State of New South Wales, Australia. The 45 and Up Study is described in detail elsewhere, ${ }^{20}$ but briefly, individuals aged 45 years and above and resident in New South Wales were randomly selected from the Medicare Australia database, which provides virtually complete coverage of the general population. Eligible individuals were mailed an invitation to take part, an information leaflet, the study questionnaire and consent form and a paid reply envelope (available at http:// www.45andUp.org.au). Participants joined the 45 and Up Study by completing the questionnaire and consent form and mailing them to the Study coordinating centre. The study oversampled, by a factor of two, individuals aged 80 years and above and people resided in rural areas; all residents of remote areas were sampled. The 45 and Up Study sample included approximately $10 \%$ of the general population in the target age range. Recruitment began in February 2006 and the analyses reported in this paper relate to the 266848 participants joining the study at the close of December 2009. The overall response rate to the mailed invitations to join the study is estimated to be $17.9 \%$; however, the exact response rate is difficult to specify as some people may not have received the invitation if their address details were incorrect in the Medicare Australia database. ${ }^{20}$ The 45 and Up Study sample has excellent heterogeneity and-in comparison to the (State of) New South Wales Population Health Survey-is reasonably representative of the New South Wales population in terms of gender, age and education; although there were differences in terms of primary language, health insurance, smoking status, psychological distress and diagnosis of some health conditions. ${ }^{21}$ Further, the study has a response rate comparable to similar studies internationally and in Australia, and is among the most representative large-scale cohort studies in the world. ${ }^{21}$ The 45 and Up Study received ethics approval from the University of New South Wales Human Research Ethics Committee.

\section{Use of $\Omega-3$}

Participants were defined as being a w3 FA supplement user if they answered 'yes' to the following question: 'In the past 4 weeks have you taken fish oil or Omega-3.'

\section{Demographic measures}

Area of residence was assigned according to the Accessibility Remoteness Index of Australia Plus score for each participant's postcode. Participants were asked about their current marital status, highest educational qualification they had completed, annual household income and their level of healthcare insurance.

\section{Health status measures}

Participants were asked to rate their overall health and overall quality of life on a five-point Likert scale. They were also asked about their history of smoking and amount of alcohol consumption. Participants were provided with a list of diseases (eg, osteoarthritis, osteoporosis, asthma, cancer) and asked if they had been treated for any of the disease in the last month. A positive response to this question for a particular disease was used to determine if a participant had that disease.

\section{Statistical analyses}

The demographic and health status characteristics of $\Omega-3$ users and non-users were compared using $\chi^{2}$ tests. 
The $\chi^{2}$ tests were used to identify those variables to be included in the logistic regression model building. Logistic regression modelling, that started with significant demographic and health characteristics (identified in the $\chi^{2}$ tests), was conducted using a backward stepwise method, to parsimoniously predict use of w3 FA supplements. In response to the large sample size and multiple comparisons, a $p$ value $<0.001$ was adopted for statistical significance. All analyses were conducted using the statistical software SAS V.9.2.

\section{RESULTS}

There were 266846 participants who answered the question regarding consumption of w3 FA supplements, of which 86939 (32.6\%) indicated that they had taken w3 FA supplements in the 4 weeks prior to the survey.

Table 1 reports demographic characteristics of participants by w3 FA supplement use. There are statistically significant associations between w3 FA supplement use and gender, age, place of residence, education, household income, marital status and health insurance (all $\mathrm{p}<0.0001$ ).

Table 2 shows health status characteristics of participants by w3 FA supplement use. There are statistically significant associations between w3 FA supplement use and smoking status, alcohol consumption, overall health, overall quality of life, osteoarthritis, asthma, cancer, high blood pressure, high cholesterol, thyroid problems, anxiety and depression (all $\mathrm{p}<0.0001$ ).

The result of the multiple logistic regression modelling is presented in table 3. Of all the diseases examined osteoarthritis, osteoporosis, high cholesterol and anxiety and/or depression were positively associated with the use of w3 FA supplements, while cancer and high blood pressure were negatively associated with the use of $\Omega-3$. That is, the odds of w3 FA supplement use was 1.65 (99\% CI 1.55 to 1.76$)$ times greater for those participants reporting treatment for osteoarthritis compared with those without osteoarthritis. The odds of $\Omega-3$ use was 1.09 (99\% CI 1.01 to 1.18 ) times greater for those

Table 1 Demographic characteristic of people aged 45 years and older by w3 FA supplement use

\begin{tabular}{|c|c|c|c|}
\hline \multirow[b]{2}{*}{ Demographic characteristics } & \multicolumn{2}{|c|}{ Use of w3 FA supplements } & \multirow[b]{2}{*}{ p Value } \\
\hline & Yes ( $n=86939) \%(S E)$ & No $(n=179907) \%(S E)$ & \\
\hline \multicolumn{4}{|l|}{ Sex } \\
\hline Female & $60(0.2)$ & $51(0.1)$ & \multirow[t]{2}{*}{$<0.0001$} \\
\hline Male & $40(0.2)$ & $49(0.1)$ & \\
\hline \multicolumn{4}{|l|}{ Age (years) } \\
\hline $45-49$ & $10(0.1)$ & $14(0.1)$ & \multirow[t]{5}{*}{$<0.0001$} \\
\hline $50-59$ & $32(0.2)$ & $34(0.1)$ & \\
\hline $60-69$ & $32(0.2)$ & $26(0.1)$ & \\
\hline $70-79$ & $17(0.1)$ & $15(0.1)$ & \\
\hline $80+$ & $9(0.1)$ & $11(0.1)$ & \\
\hline \multicolumn{4}{|l|}{ Place of residence } \\
\hline Major city & $45(0.2)$ & $45(0.1)$ & \multirow[t]{4}{*}{$<0.0001$} \\
\hline Inner regional & $36(0.2)$ & $35(0.1)$ & \\
\hline Outer regional & $17(0.1)$ & $18(0.1)$ & \\
\hline Remote/very remote & $2(0.1)$ & $2(0.1)$ & \\
\hline \multicolumn{4}{|l|}{ Education } \\
\hline School certificate or less & $34(0.2)$ & $34(0.1)$ & \multirow[t]{4}{*}{0.0001} \\
\hline Higher school certificate & $10(0.1)$ & $10(0.1)$ & \\
\hline Trade/certificate/diploma & $33(0.2)$ & $32(0.1)$ & \\
\hline Tertiary & $23(0.1)$ & $24(0.1)$ & \\
\hline \multicolumn{4}{|l|}{ Annual } \\
\hline$<\$ 20000$ & $25(0.1)$ & $25(0.1)$ & \multirow[t]{5}{*}{$<0.0001$} \\
\hline \multicolumn{3}{|l|}{ Household income } & \\
\hline$\$ 20000-\$ 49999$ & $33(0.2)$ & $31(0.1)$ & \\
\hline$\$ 50000-\$ 69999$ & $14(0.1)$ & $13(0.1)$ & \\
\hline$\geq \$ 70000$ & $28(0.2)$ & $31(0.1)$ & \\
\hline \multicolumn{4}{|l|}{ Marital status } \\
\hline Married/defacto & $75(0.1)$ & $75(0.1)$ & \multirow[t]{3}{*}{$<0.0001$} \\
\hline Widow/divorce/separ. & $20(0.1)$ & $19(0.1)$ & \\
\hline Single & $5(0.1)$ & $6(0.1)$ & \\
\hline \multicolumn{4}{|l|}{ Health } \\
\hline Private & $55(0.2)$ & $53(0.1)$ & \multirow[t]{4}{*}{$<0.0001$} \\
\hline \multicolumn{3}{|l|}{ Insurance } & \\
\hline DVA or HCC & $30(0.2)$ & $29(0.1)$ & \\
\hline None & $15(0.1)$ & $18(0.1)$ & \\
\hline
\end{tabular}


Table 2 Health status characteristics of people aged 45 years and older by w3 FA supplement use

\begin{tabular}{|c|c|c|c|}
\hline \multirow[b]{2}{*}{ Health status characteristics } & \multicolumn{2}{|c|}{ Use of w3 FA supplements } & \multirow[b]{2}{*}{ p Value } \\
\hline & Yes (n=86939) \% (SE) & No $(n=179907) \%$ (SE) & \\
\hline \multicolumn{4}{|l|}{ Smoking status } \\
\hline Current smoker & $5(0.1)$ & $8(0.1)$ & $<0.0001$ \\
\hline Former smoker & $36(0.2)$ & $35(0.1)$ & \\
\hline Never smoked & $59(0.2)$ & $56(0.1)$ & \\
\hline \multicolumn{4}{|l|}{ Alcohol } \\
\hline 0-6 drinks/week & $64(0.2)$ & $62(0.1)$ & $<0.0001$ \\
\hline \multicolumn{4}{|l|}{ Consumption } \\
\hline 7-13 drinks/week & $19(0.1)$ & $19(0.1)$ & \\
\hline 14-20 drinks/week & $11(0.1)$ & $11(0.1)$ & \\
\hline$\geq 21$ drinks/week & $6(0.1)$ & $8(0.1)$ & \\
\hline \multicolumn{4}{|l|}{ Overall health } \\
\hline Excellent/very good/good & $87(0.1)$ & $85(0.1)$ & $<0.0001$ \\
\hline Fair/poor & $13(0.1)$ & $15(0.1)$ & \\
\hline \multicolumn{4}{|l|}{ Overall quality } \\
\hline Excellent/very good/good & $90(0.1)$ & $89(0.1)$ & $<0.0001$ \\
\hline \multicolumn{4}{|l|}{ Of life } \\
\hline Fair/poor & $10(0.1)$ & $11(0.1)$ & \\
\hline \multicolumn{4}{|l|}{ Osteoarthritis } \\
\hline Yes & $11(0.1)$ & $7(0.1)$ & $<0.0001$ \\
\hline No & $89(0.1)$ & $93(0.1)$ & \\
\hline \multicolumn{4}{|l|}{ Osteoporosis } \\
\hline Yes & $7(0.1)$ & $5(0.1)$ & $<0.0001$ \\
\hline No & $93(0.1)$ & $95(0.1)$ & \\
\hline \multicolumn{4}{|l|}{ Asthma } \\
\hline Yes & $5(0.1)$ & $4(0.1)$ & $<0.0001$ \\
\hline No & $95(0.1)$ & $96(0.1)$ & \\
\hline \multicolumn{4}{|l|}{ Cancer } \\
\hline Yes & $2(0.1)$ & $3(0.1)$ & $<0.0001$ \\
\hline No & $98(0.1)$ & $97(0.1)$ & \\
\hline \multicolumn{4}{|l|}{ High blood pressure } \\
\hline Yes & $26(0.1)$ & $24(0.1)$ & $<0.0001$ \\
\hline No & $74(0.1)$ & $76(0.1)$ & \\
\hline \multicolumn{4}{|l|}{ High cholesterol } \\
\hline Yes & $17(0.1)$ & $14(0.1)$ & $<0.0001$ \\
\hline No & $83(0.1)$ & $86(0.1)$ & \\
\hline \multicolumn{4}{|l|}{ Heart attack or angina } \\
\hline Yes & $3(0.1)$ & $3(0.1)$ & 0.2996 \\
\hline No & $97(0.1)$ & $97(0.1)$ & \\
\hline \multicolumn{4}{|l|}{ Other heart disease } \\
\hline Yes & $3(0.1)$ & $3(0.1)$ & 0.6606 \\
\hline No & $97(0.1)$ & $97(0.1)$ & \\
\hline \multicolumn{4}{|l|}{ Thyroid problems } \\
\hline Yes & $6(0.1)$ & $5(0.1)$ & $<0.0001$ \\
\hline No & $94(0.1)$ & $95(0.1)$ & \\
\hline \multicolumn{4}{|l|}{ Anxiety and depression } \\
\hline Neither & $91(0.1)$ & $92(0.1)$ & $<0.0001$ \\
\hline Depression only & $4(0.1)$ & $4(0.1)$ & \\
\hline Anxiety only & $2(0.1)$ & $1(0.1)$ & \\
\hline Both & $3(0.1)$ & $3(0.1)$ & \\
\hline
\end{tabular}

participants reporting osteoporosis compared with those without osteoporosis. In comparison to participants reporting treatment for anxiety or depression, those participants with anxiety only or both anxiety and depression were 1.16 (99\% CI 1.01 to 1.34$)$ and 1.19 (99\% CI
1.07 to 1.32 ) times more likely to use w3 FA supplements, respectively. Those participants reporting treatment for high cholesterol were 1.23 (99\% CI 1.17 to 1.29) times more likely to use $\Omega-3$ compared with those without high cholesterol. The odds of w3 FA 
Table 3 Multiple logistic regression model for predicting use of w3 FA supplements in people aged 45 years and older

\begin{tabular}{|c|c|c|}
\hline Factor & OR & $99 \% \mathrm{Cl}$ \\
\hline \multicolumn{3}{|l|}{ Sex } \\
\hline Male & 1.00 & - \\
\hline Female & 1.42 & 1.37 to 1.48 \\
\hline \multicolumn{3}{|l|}{ Age } \\
\hline $45-49$ & 1.00 & - \\
\hline $50-59$ & 1.43 & 1.36 to 1.52 \\
\hline $60-69$ & 1.84 & 1.73 to 1.96 \\
\hline $70-79$ & 1.76 & 1.64 to 1.90 \\
\hline $80+$ & 1.32 & 1.21 to 1.44 \\
\hline \multicolumn{3}{|l|}{ Place of residence } \\
\hline Major city & 1.00 & - \\
\hline Inner regional & 0.98 & 0.95 to 1.02 \\
\hline Outer regional & 0.89 & 0.85 to 0.93 \\
\hline Remote/very remote & 0.86 & 0.75 to 0.98 \\
\hline \multicolumn{3}{|l|}{ Annual } \\
\hline$<\$ 20000$ & 1.00 & - \\
\hline \multicolumn{3}{|l|}{ Household income } \\
\hline$\$ 20000-\$ 49999$ & 1.14 & 1.08 to 1.20 \\
\hline$\$ 50000-\$ 69999$ & 1.13 & 1.06 to 1.21 \\
\hline$\geq \$ 70000$ & 1.03 & 0.97 to 1.10 \\
\hline \multicolumn{3}{|l|}{ Insurance } \\
\hline Private & 1.00 & - \\
\hline DVA or HCC & 0.93 & 0.89 to 0.98 \\
\hline None & 0.84 & 0.80 to 0.89 \\
\hline \multicolumn{3}{|l|}{ Smoking status } \\
\hline Current smoker & 1.00 & - \\
\hline Former smoker & 1.66 & 1.53 to 1.79 \\
\hline Never smoked & 1.56 & 1.44 to 1.69 \\
\hline \multicolumn{3}{|l|}{ Alcohol } \\
\hline 0-6 drinks/week & 1.00 & - \\
\hline \multicolumn{3}{|l|}{ Consumption } \\
\hline 7-13 drinks/week & 1.02 & 0.97 to 1.07 \\
\hline 14-20 drinks/week & 0.94 & 0.89 to 1.00 \\
\hline$\geq 21$ drinks/week & 0.83 & 0.78 to 0.89 \\
\hline \multicolumn{3}{|l|}{ Overall health } \\
\hline Excellent/very good/good & 1.00 & - \\
\hline Fair/poor & 0.82 & 0.77 to 0.86 \\
\hline \multicolumn{3}{|l|}{ Osteoarthritis } \\
\hline No & 1.00 & - \\
\hline Yes & 1.65 & 1.55 to 1.76 \\
\hline \multicolumn{3}{|l|}{ Osteoporosis } \\
\hline No & 1.00 & - \\
\hline Yes & 1.09 & 1.01 to 1.18 \\
\hline \multicolumn{3}{|l|}{ Cancer } \\
\hline No & 1.00 & - \\
\hline Yes & 0.89 & 0.80 to 0.99 \\
\hline \multicolumn{3}{|l|}{ High blood pressure } \\
\hline No & 1.00 & - \\
\hline Yes & 0.95 & 0.91 to 0.99 \\
\hline \multicolumn{3}{|l|}{ High cholesterol } \\
\hline No & 1.00 & - \\
\hline Yes & 1.23 & 1.17 to 1.29 \\
\hline \multicolumn{3}{|l|}{ Anxiety and depression } \\
\hline Neither & 1.00 & - \\
\hline Depression only & 1.01 & 0.92 to 1.11 \\
\hline Anxiety only & 1.16 & 1.01 to 1.34 \\
\hline Both & 1.19 & 1.07 to 1.32 \\
\hline
\end{tabular}

supplement use was 0.89 (99\% CI 0.80 to 0.99$)$ and 0.95 (99\% CI 0.91 to 0.99 ) times lower for those participants reporting treatment for cancer and high blood pressure, respectively.

Table 3 also shows that those participants who rated their overall health to be fair or poor were 0.82 (99\% CI 0.77 to 0.86 ) less likely to use $\Omega-3$. In comparison to current smokers, participants who were former smokers $(\mathrm{OR}=1.66 ; 99 \%$ CI 1.53 to 1.79$)$ or never smoked $(\mathrm{OR}=1.56 ; 99 \%$ CI 1.44 to 1.69$)$ were more likely to use w3 FA supplements. In comparison with those participants who drank 0-6 alcoholic drinks per week, participants who drank 14-20 alcoholic drinks $(\mathrm{OR}=0.94 ; 99 \%$ CI 0.89 to 1.00$)$ or $\geq 21$ alcoholic drinks (OR=0.83; $99 \%$ CI 0.78 to 0.89 ) were less likely to use w3 FA supplements. Participants with no health insurance were 0.84 (99\% CI 0.80 to 0.89 ) times less likely to use w3 FA supplements compared with participants with private health insurance. In terms of household income, the odds of w3 FA supplement use were 1.14 (99\% CI 1.08 to 1.20$)$ and 1.13 (99\% CI 1.06 to 1.21) greater for participants with an income of \$20000-\$49 999 and \$50000$\$ 69999$, respectively, compared with those with an income of $<\$ 20000$. In comparison to those participants who live in a major city, the odds of w3 FA supplement use are less for those living in outer regional areas $(\mathrm{OR}=0.89 ; 99 \%$ CI 0.85 to 0.93$)$ and remote or very remote areas $(\mathrm{OR}=0.86 ; 99 \%$ CI 0.75 to 0.98$)$. In comparison with those participants aged 45-49 years, all other age groups have greater odds of w3 FA supplement use, with the highest being those aged 60-69 $(\mathrm{OR}=1.84 ; 99 \%$ CI 1.73 to 1.96$)$ and $70-79(\mathrm{OR}=1.76$; $99 \%$ CI 1.64 to 1.90$)$ years. In terms of gender, the odds of w3 FA supplement use was 1.42 (99\% CI 1.36 to 1.52$)$ times greater for female participants.

\section{DISCUSSION}

Our study, drawing upon the largest database with regard to w3 FA supplement use to date and constituting the first analyses of the profile of users and prevalence of use of w3 FA supplements in Australia, shows $32.6 \%$ of the study participants, aged 45 years and above, consume w3 FA supplements. This finding identifies w3 FA supplements as one of the most commonly used dietary supplements among older Australians and is in line with a previous research showing w3 FA supplements as among the top five complementary and alternative medicines recommended by Australian GPs and community pharmacists. ${ }^{4}$ The discovery of such a high level of w3 FA supplement use among older Australians suggests that further research is needed to explore consumer behaviours and decision-making regarding w3 FA supplement use alongside assessing the possible health impacts of such a consumption.

Overall, the findings of association between being woman, of increased age, having advanced education and higher use of w3 FA supplements are congruent 
with factors predicting broader complementary and alternative medicine use. ${ }^{3}{ }^{22}$ The association of w3 FA supplement use with higher annual income and private health insurance highlights the potential importance of cost of w3 FA supplement products with regard to consumption and this issue warrants further investigation. It is also important to note that w3 FA supplements, like many complementary and alternative medicine products more generally, is not currently subsidised by the Australian Pharmaceutical Benefits Scheme (a Federal government programme providing subsidised prescription drugs to residents) and attracts a further $10 \%$ goods and services tax that prescription medicines do not. As such, cost issues associated with w3 FA supplementation may be more focused in our Australian population. Our finding of a positive association of w3 FA supplement use with higher annual income may also relate to the suggestion that socioeconomic status acts as a protective factor in health, with those having better life chances more likely to adopt self-care measures to maintain their health and quality of life. ${ }^{23}$

The low use of w3 FA supplements among participants resident in rural and remote areas compared with those respondents living in metropolitan locations contradicts the findings of a previous research which shows higher CAM use in rural areas in Australia. ${ }^{24}$ However, it does reflect findings from some national and international studies which suggest lower rates of commercial or prepackaged CAM product use among some rural populations, when compared with their urban counterparts, which may be associated with reduced access to these supplements. ${ }^{24}$ Indeed, the urban-rural divide in the use of complementary and alternative medicine is an issue that has received much attention in recent years ${ }^{24-26}$ and the results from our study help add to the evidence-base and discussion of this important health service issue and highlight the need for further investigation into the complexities of regional variation in supplement use.

The variations in the association of w3 FA supplement use with a range of clinical conditions are noteworthy, especially given the current varied clinical evidence-base of w3 FA supplements. The finding of low w3 FA supplement use among people with cancer is not unexpected as research evidence suggests no association between w3 FA supplements and reducing cancer incidence. ${ }^{10}$ Additionally, external factors may also result in lower $\Omega-3$ supplement use among people with cancer: patients may relinquish their CAM use when their use of other forms of medical treatment increases ${ }^{27}$; or patients may be advised to cease all other medications when undergoing cancer treatment. ${ }^{28}$ However, it is somewhat surprising that high blood pressure is negatively associated with the use of w3 FA supplement as there is evidence that $\Omega-3$ has beneficial effects in the context of cardiovascular disease and in lowering blood pressure. ${ }^{29}$ This is an area worthy of further empirical investigation.

The association of higher w3 FA supplement use with diseases such as osteoarthritis, osteoporosis, anxiety and/or depression is interesting given there is currently either conflicting or insufficient evidence on the efficacy of W3 FA supplements in addressing these conditionsthis suggests there may currently exist a mismatch between clinical evidence and consumers' perceptions of evidence and benefits regarding w3 FA supplement use. Together, these study findings highlight the potential need for nutrition guidelines for w3 FA supplement intake and consumer awareness of the use of w3 FA supplements as well as possible enhanced information and labelling of relevant products in Australia. The study findings also add weight to recently identified evidence illustrating a desire for good-quality information about w3 FA supplement products among GPs and pharmacists. ${ }^{4}$

The finding that respondents with better quality of life/health ratings or a healthy lifestyle (eg, non-smoking and/or having less alcoholic drinks) have greater odds of w3 FA supplement use may indicate that w3 FA supplements are used for both the treatment of specific health conditions and as a preventive therapy. Previous research suggests that this distinction between therapeutic and preventive use also exists for complementary and alternative medicine consumption more broadly. ${ }^{30}$ Given these results, it would be useful for future studies to differentiate between these two approaches (therapeutic and preventive use) to use and to provide critical, in-depth examination of patients' motivations and understandings regarding consumption of w3 FA supplements and other CAM or dietary supplements.

The interpretation of our findings is limited by the fact that the association between w3 FA supplement consumption with particular health conditions does not necessarily imply that w3 FA supplements have been used specifically for these conditions. In addition, the disease variable used from the 45 and Up Study survey was based on individuals reporting that they had been 'treated in last month' rather than 'ever been diagnosed' and that health, w3 FA supplement use and healthcare use is selfreported by the participants. As such our study results may be subject to recall bias and we may have missed some participants who had a disease but were not treated for it in the month prior to being surveyed. Currently, this study focuses solely on the use of W3 FA supplements, and this research may have benefitted by including an analysis of usual dietary intake (eg, food frequency questionnaire), in particular the consumption of $\Omega$-3-rich foods such as oily fish that may be also be consumed for therapeutic benefit. Given the sample of 45 and Up Study was drawn from the State of New South Wales, generalisation of the findings of this research to other parts of Australia should be treated with caution and as the study sample has been shown to be not representative of the New South Wales population on a number of characteristics, caution should be made in generalising the findings to the New South Wales population. Finally, as the statistical tests used in our analyses are influenced by sample size, the very large sample size in this study can make small differences appear to be significant. As such, 
readers need to take into account the absolute differences while interpreting the ORs. Nevertheless, these limitations are countered by the insights gained from analysing data from the largest sample of adults aged 45 years and above with regard to their consumption of w3 FA supplements.

\section{CONCLUSION}

Our analysis of data from the 45 and Up Study cohort suggests that W3 FA supplements are consumed for a wide variety of purposes by a considerable proportion of Australians aged 45 years and above. In the context of these study findings there is a need for primary healthcare practitioners to enquire with their patients about their use of w3 FA supplements as well as for further work to ensure provision of good-quality information for patients and providers with regard to w3 FA products.

Acknowledgements We thank the men and women participating in the 45 and Up Study. The 45 and Up Study is managed by the Sax Institute in collaboration with major partner Cancer Council New South Wales, and partners: the National Heart Foundation of Australia (NSW Division); NSW Health; beyondblue: the national depression initiative; Ageing, Disability and Home Care, Department of Human Services NSW and UnitingCare Ageing.

Contributors All authors devised the study and helped conceptualise ideas, interpret findings, manuscript writing and reviewing drafts of the article. JA and DS acquired the data from the 45 and Up Study. DS undertook data analysis and JA, DS, C-WL, AB and JW contributed to the interpretation of the data. All authors read and approved the final manuscript.

Funding This research received no specific grant from any funding agency in the public, commercial or not-for-profit sectors.

Competing interests None.

Provenance and peer review Not commissioned; externally peer reviewed.

Data sharing statement No additional data are available.

\section{REFERENCES}

1. Gahche J, Bailey R, Burt V, et al. Dietary supplement use among USA adults has increased since NHANES III (1988-1994). NCHS Data Brief. Atlanta: Centers for Disease Control and Prevention, 2011:1-8.

2. Skeie G, Braaten T, Hjartåker A, et al. Use of dietary supplements in the European Prospective Investigation into Cancer and Nutrition calibration study. Eur J Clin Nutr 2009;63:226-38.

3. Barnes PM, Bloom B, Nahin RL. Complementary and alternative medicine use among adults and children: United States, 2007. Hyattsville: U.S. Department of Health and Human Services, Division of Health Interview Statistics, Centers for Disease Control and Prevention, National Center for Health Statistics, 2008.

4. Brown J, Morgan T, Adams J, et al. Complementary medicines information use and needs of health professionals: general practitioners and pharmacists. Sydney: National Prescribing Service, 2008

5. Chowdhury R, Stevens S, Gorman D, et al. Association between fish consumption, long chain omega 3 fatty acids, and risk of cerebrovascular disease: systematic review and meta-analysis. $B M J$ 2012;345:e6698.

6. Filion KB, El Khoury F, Bielinski M, et al. Omega-3 fatty acids in high-risk cardiovascular patients: a meta-analysis of randomized controlled trials. BMC Cardiovasc Disord 2010;10.

7. Kromhout D, Yasuda S, Geleijnse JM, et al. Fish oil and omega-3 fatty acids in cardiovascular disease: do they really work? Eur Heart J 2012;33:436-43.
8. Rizos EC, Ntzani EE, Bika E, et al. Association between omega-3 fatty acid supplementation and risk of major cardiovascular disease events: a systematic review and meta-analysis. JAMA 2012;308:1024-33.

9. National Heart Foundation of Australia. Position statement: fish, fish oils, $n-3$ polyunsaturated fatty acids and cardiovascular health. Sydney: National Heart Foundation of Australia, 2008.

10. MacLean $\mathrm{CH}$, Newberry SJ, Mojica WA, et al. Effects of omega-3 fatty acids on cancer risk: a systematic review. JAMA 2006;295:403-15.

11. Colomera R, Moreno-Nogueiraa JM, García-Lunaa PP, et al. N-3 fatty acids, cancer and cachexia: a systematic review of the literature. Br J Nutr 2007;97:823-31.

12. Lin P-Y, Su K-P. A meta-analytic review of double-blind, placebo-controlled trials of antidepressant efficacy of Omega-3 fatty acids. J Clin Psychiatry 2007;68:1056-61.

13. Montgomery $\mathrm{P}$, Richardson AJ. Omega-3 fatty acids for bipolar disorder. Cochrane Database Syst Rev 2008;2:CD005169. doi: 10.1002/14651858.CD005169.pub2

14. Thien FCK, De Luca S, Woods RK, et al. Dietary marine fatty acids (fish oil) for asthma in adults and children. Cochrane Database Syst Rev 2003;3:CD001283. doi: 10.1002/14651858.CD001283

15. Oliver C, Everard M, N'Diaye T. Omega-3 fatty acids (from fish oils) for cystic fibrosis. Cochrane Database Syst Rev 2007;4: CD002201.

16. Agency for Healthcare Research and Quality. Effects of omega-3 fatty acids on lipids and glycemic control in type II diabetes and the metabolic syndrome and on inflammatory bowel disease, rheumatoid arthritis, renal disease, systemic lupus erythematosus, and osteoporosis. Rockville: Agency for Healthcare Research and Quality, 2004

17. Sydenham E, Dangour AD, Lim W-S. Omega 3 fatty acid for the prevention of cognitive decline and dementia. Cochrane Database Syst Rev 2012;6:CD005379. doi: 10.1002/14651858.CD005379.

18. James S, Montgomery P, Williams K. Omega-3 fatty acids supplementation for autism spectrum disorders (ASD). Cochrane Database Syst Rev 2011;11:CD007992. doi: 10.1002/14651858. CD007992.

19. Kickbusch I, Payne L. Twenty-first century health promotion: the public health revolution meets the wellness revolution. Health Promot Int 2003;18:275-8.

20. 45 and Up Study Collaborators. Cohort profile: the 45 and Up Study. Int J Epidemiol 2008;37:941-7.

21. Mealing N, Banks E, Jorm L, et al. Investigation of relative risk estimates from studies of the same population with contrasting response rates and designs. BMC Med Res Methodol 2010;10.

22. Adams J, Sibbritt D, Easthope G, et al. The profile of women who consult alternative health practitioners in Australia. Med $J$ Aust 2003:179:297-300.

23. Cockerham WC. Health lifestyles: bringing structure back. In: Cockerham WC, ed. The new Blackwell companion to medical sociology. Oxford: Wiley-Blackwell, 2009:159-83.

24. Wardle J, Lui C-W, Adams J. Complementary and alternative medicine in rural communities: current research and future directions. J Rural Health 2012;28:101-12.

25. Adams J, Sibbritt D, Lui C-W. The urban-rural divide in complementary and alternative medicine use: a longitudinal study of 10,638 women. BMC Complement Altern Med 2011;11:2.

26. Adams J. Exploring the interface between complementary and alternative medicine (CAM) and rural general practice: $A$ call for research. Health Place 2004;10:285-87.

27. Sibbritt DW, Adams J, Young AF. A longitudinal analysis of mid-age women's use of complementary and alternative medicine (CAM) in Australia, 1996-1998. Women Health 2004:40:41-56.

28. Broom A, Adams J. Oncology clinicians' accounts of discussing complementary and alternative medicine with their patients. Health 2009;13:317-36.

29. Wang C, Harris WS, Chung M, et al. N-3 fatty acids from fish or fish-oil supplements, but not alpha-linolenic acid, benefit cardiovascular disease outcomes in primary- and secondary-prevention studies: a systematic review. Am J Clin Nutr 2006;84:5-17.

30. Bishop FL, Yardley L, Lewith GT. Treat or treatment-a qualitative study analyzing patients' use of CAM. Am J Public Health 2008;98:1700-5. 